\title{
Papillary Thyroid Cancer with Synchronous Lymph Nodes Metastases: Left Lateral Neck Dissection and Total Thyroidectomy with Continuous Intraoperative Nerve
}

Maria G. Chiofalo, MD

Thyroid Surgery Unit, Istituto Nazionale Tumori, IRCCS Fondazione G. Pascale, Naples, Italy. E-mail:m.chiofalo@istitutotumori.na.it

Luciano Pezzullo, MD

Thyroid Surgery Unit, Istituto Nazionale Tumori, IRCCS Fondazione G. Pascale, Naples, Italy.

Presented at the 3rd World Congress on Thyroid Cancer, July 29, 2017.

(c) Mary Ann Liebert, Inc. and American Thyroid Association DOI: 10.1089/ve.2018.0149
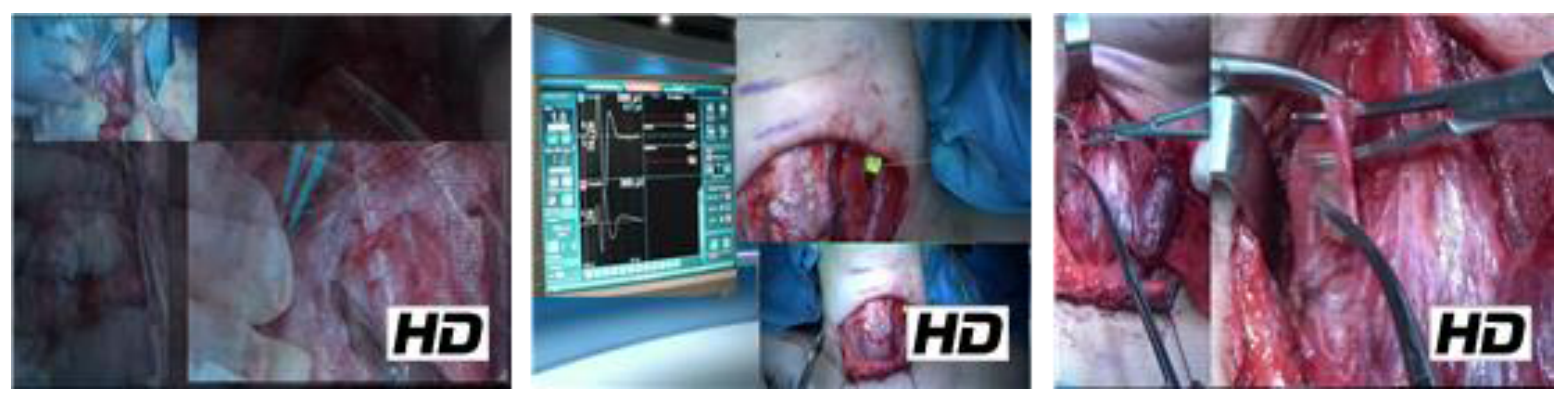

\section{Abstract}

Introduction: Lymph node metastases from papillary thyroid cancer are common. According to American Thyroid Association thyroid cancer guidelines, "lateral neck dissection should be performed only as a therapeutic intervention for known disease." Intraoperative neuromonitoring (IONM) has been introduced as an adjunct to routine visual identification of the recurrent laryngeal nerve during thyroid surgery. IONM is helpful in early identification of the recurrent laryngeal nerve (RLN) and its anatomical variants; by prognosticating postoperative nerve function, it led to prevent bilateral recurrent laryngeal nerve lesions. ${ }^{2,3}$ Continuous intraoperative nerve monitoring (C-IONM) is an evolution of neuromonitoring that provides real-time evaluation of the RLN function during thyroid surgery. By detecting early electromiography (EMG) changes, it may identify impending nerve injury, allowing promptly corrective surgical maneuvers to protect the RLN integrity. ${ }^{4,5}$

Case: This video shows the surgical treatment of a papillary thyroid carcinoma metastatic to the left lateral neck in a 25-year-old woman. A selective lateral neck dissection (levels II-IV) and a near total thyroidectomy with intraoperative nerve monitoring were performed. The intraoperative nerve monitoring was performed with the standardized technique according to the recommendations of the International Intraoperative Neural Monitoring Study Group (L1+V1+R1+R2+V2+L2). External branch of the superior laryngeal nerve (EBSLN) was also identified and monitored. On the left side, real-time C-IONM was performed by placing an automatic periodic stimulation probe over the vagus nerve, whereas a stimulator probe delivering intermittent electrical current was used for identification and mapping of the RLN and EBSLN during the entire surgical procedure. At the end of the left thyroid lobe dissection, before proceeding to the right thyroid lobe dissection, the left recurrent laryngeal nerve and vagus nerve were checked. The right thyroid lobe dissection was performed only with intermittent neuromonitoring. In the right side, the RLN was branched, by using IONM, it was possible to identify the motor branch of the RLN, which was the anterior one. The IONM is useful in cases like this to avoid nerve injury; missing a branched nerve is the main reason for nerve injury. 
Conclusion: IONM is useful to facilitate intraoperative nerve identification and to provide valuable information on nerve function after dissection. Continuous IONM may prevent nerve injury by detecting intraoperative EMG changes that indicate impending RLN injury. To avoid bilateral nerve injury it is crucial to perform intraoperative nerve monitoring with the standardized technique according to the recommendations of the International Intraoperative Neural Monitoring Study Group, by checking the vagus nerve at the end of the dissection and before proceeding to the contralateral site excision. The goal of the management of papillary thyroid cancer metastatic to the lateral neck is the removal of macroscopic disease at the time of initial surgery. Appropriate surgical treatment should balance the known risks of lateral neck dissection against the indolent behavior of differentiated thyroid cancer. Optimizing the surgical approach is fundamental to ensure the appropriate treatment of differentiated thyroid cancer metastatic to the lateral neck lymph nodes.

No competing financial interests exist.

Runtime of video: 7 mins 45 secs

Presented at the 3rd World Congress on Thyroid Cancer, July 29, 2017.

Keywords: papillary thyroid cancer, intraoperative nerve monitoring, lymphnode metastases

\section{Cite this video}

Maria G. Chiofalo, Luciano Pezzullo, Papillary Thyroid Cancer with Synchronous Lymph Nodes Metastases: Left Lateral Neck Dissection and Total Thyroidectomy with Continuous Intraoperative Nerve, VideoEndocrinology. 2019, DOI: 10.1089/ve.2018.0149.

\section{References}

1. Haugen BR, Alexander EK, Bible KC, et al. 2015 American Thyroid Association management guidelines for adult patients with thyroid nodules and differentiated thyroid cancer: The American Thyroid Association guidelines task force on thyroid nodules and differentiated thyroid cancer. Thyroid 2016;26:1-133.

2. Randolph GW, Dralle H, International Intraoperative Monitoring Study Group, et al. Electrophysiologic recurrent laryngeal nerve monitoring during thyroid and parathyroid surgery: International standards guideline statement. Laryngoscope 2011;121 Suppl 1:S1-S16.

3. Deniwar A, Kandil E, Randolph G. Electrophysiological neural monitoring of the laryngeal nerves inthyroid surgery: Review of the current literature. Gland Surg 2015;4:368-375.

4. Phelan $\mathrm{E}$, Schneider $\mathrm{R}$, Lorenz $\mathrm{K}$, et al. Continuous vagal ionm prevents recurrent laryngeal nerve paralysis by revealing initial EMG changes of impending neuropraxic injury: A prospective, multicenter study. Laryngoscope 2014;124:1498-1505.

5. Kandil E, Mohsin K, Murcy MA, Randolph GW. Continuous vagal monitoring value in prevention of vocal cord paralysis following thyroid surgery. Laryngoscope 2018;128:2429-2432.

Original Publication Date: 2017 